ISSN 2145-6054

\title{
DERECHO Y ECONOMÍA, LA FORMULA PARA LA SUPERACIÓN DE LA POBREZA EXTREMA Y LA CONSTRUCCIÓN DE PAZ EN COLOMBIA ${ }^{1}$ Law and economy: the formula to overcome extreme poverty and to build peace in Colombia
}

\author{
Georgina De León Vargas ${ }^{2}$ \\ Damary Margarita Ballestas León ${ }^{3}$
}

Fecha de recepción: 11 de abril de 2017

Fecha de aceptación: 04 de mayo de 2017

Sumario: 1. Introducción; 1.1 ¿Por qué hablar de pobreza extrema y construcción de paz en Colombia?; 1.2 Inventiva para la recolección y el análisis de información; 2. La economía, el derecho y el conflicto; 3. Desarrollo integral, más allá de la distribución económica; 4. La teoría del desarrollo en el plan nacional de desarrollo (PND); 5. La educación como eje central; 6. Conclusiones; 7. Referencias Bibliográficas.

\footnotetext{
${ }^{1}$ Avances de la investigación "El programa de Colombia equitativa y sin pobreza extrema en los municipios de San Juan Nepomuceno y el Carmen de Bolívar" adscrito al Grupo de Investigación DERECHO PÚBLICO del Programa de Derecho de la Corporación Universitaria Rafael Núñez sede Cartagena, financiado por esta entidad en Convocatoria interna 005 de 2016.

${ }^{2}$ Abogada de la Corporación universitaria Rafael Núñez. Magíster en Derecho Laboral, Universidad Autónoma Guerrero de México. Docente de tiempo completo, con funciones de coordinación en investigación del programa de derecho, Corporación Rafael Núñez sede Cartagena y adscrita al Grupo de Investigación Derecho Público de la Corporación Universitaria Rafael Núñez.

${ }^{3}$ Trabajadora Social, egresada de la universidad de Cartagena. Estudiante de V semestre de derecho, en la corporación universitaria Rafael Núñez. Miembro activa del semillero de investigación SIFAD.
} 


\section{CÓMO SE CITA ESTE ARTíCULO (Normas APA-6)}

De León Vargas, Georgina \& Ballestas León, Damary Margarita (2017). Derecho y economía: Fórmula para la superación de la pobreza extrema y la construcción de paz en Colombia. Revista Jurídica Mario Alario D’Filippo, IX (18), pág 41-54.

\section{RESUMEN}

Este artículo es un avance de un trabajo investigativo que busca analizar el plan nacional y los planes municipales de desarrollo de los municipios de El Carmen de Bolívar y San Juan Nepomuceno, para identificar las propuestas de desarrollo integral, como estrategia para la superación de la pobreza extrema y en consecuencia la construcción de paz. La pobreza no solo implica bajos niveles de ingreso; usualmente está acompañada por la vulneración del acceso de los derechos fundamentales. Reducir la pobreza requiere incentivar la generación de oportunidades para la consecución de un desarrollo económico y social. La planificación en todos los niveles es pieza fundamental, la concordancia, el trabajo articulado y transparente es en gran medida la garantía para el desarrollo integral.

\section{PALABRAS CLAVE}

Derecho, economía, desarrollo integral.

\section{ABSTRACT}

This paper is the result of a research work that seeks to analyze the national and the municipal development plans of El Carmen de Bolívar and San Juan Nepomuceno, in order to offer some proposals for their integral development, as a strategy to overcome extreme poverty and, consequently, build peace. Poverty does not only imply low levels of income, but usually comes together with fundamental rights violations. Reducing poverty requires the generation of opportunities for the achievement of economic and social development. Planning is a fundamental requirement at all levels, while concordance and articulated and transparent work is basically the guarantee for integral development.

\section{KEYWORDS}

Law, economy, integral development 


\section{INTRODUCCIÓN}

\section{1 ¿POR QUÉ HABLAR DE POBREZA EXTREMA Y CONSTRUCCIÓN DE PAZ EN COLOMBIA?}

La superación de la pobreza extrema en Colombia se ha convertido en la bandera principal de los planes nacionales de desarrollo, que plantean propuestas dirigidas a vencer el estado de vulneración de millones de colombianos. La preocupante situación de poblaciones específicas como los campesinos, con sus problemas comunes como la falta de tierra, la carencia de maquinarias para la producción cuando las condiciones económicas exigen un campo industrializado, la no garantía de sus derechos, y en general la poca protección a la vida ha hecho que los distintos gobiernos estén obligados a pensar estrategias para vencer la pobreza extrema. Sin embargo, factores como el conflicto interno han sido otrora un restrictivo hecho para el avance.

El actual plan nacional de desarrollo: Todos por un nuevo país, que cobija los años 20142018, propone en su lema: paz, equidad y educación; planteando estos, como estrategia principal en sus líneas contra la pobreza. Sin embargo, la coyuntura política del proceso de paz, donde los principales actores del conflicto armado colombiano están a un paso de regresar a la vida civil, no permite dejar por fuera la construcción de paz, puesto que la guerra ha sido a lo largo de la historia un limitante de la inversión estatal en los territorios.

La coyuntura política, económica y social que vive el país, exige que se analice desde la disciplina de la economía y el derecho como dúo fundamental para abordar el desarrollo integral, puesto que la pobreza más que un fenómeno económico, es, en el más complejo de los casos un problema socio-económico que sin duda, involucra al derecho como herramienta para la exigibilidad del objetivo de la economía, que no es otro que la distribución de los bienes, sin embargo ¿Qué sucede con las relaciones sociales cuando tales bienes son distribuidos de forma inequitativa? Es aquí donde entra a accionar el derecho.

Por lo anterior, es de carácter relevante dar lugar a la comparación de las situaciones de pobreza-desigualdad en los estados de guerra o de conflicto, como es el caso de Colombia, analizando la relación de los programas Colombia equitativa y sin pobreza extrema y Colombia en paz, del plan nacional de desarrollo 2014-2018, como los dos ejes fundamentales para el desarrollo integral de los territorios.

\subsection{INVENTIVA PARA LA RECOLECCIÓN Y EL ANÁLISIS DE INFORMACIÓN}

Analizar los programas Colombia en paz y Colombia equitativa y sin pobreza extrema del Plan Nacional de Desarrollo todos por un nuevo país 2014-2018, exige el contraste con teorías económicas demostradas sostenibles, por ello, la teoría del desarrollo integral del 
economista Amatya Sen, se examinará en primera instancia con las propuestas del plan nacional de desarrollo, y en segunda frente a la materialización de las apuestas políticas en los municipios de San Juan Nepomuceno y el Carmen de Bolívar.

Se acudió a la metodología de tipo explicativo-experimental, puesto que fue necesario adentrarse en terreno para explicar los fenómenos que ocurren en la realidad, así como para determinar su origen y causas. Desde lo experimental, es posible plantear la causaefecto de la problemática.

Es de carácter socio-jurídica, puesto que interpela "los espacios de jurídica, los saberes jurídicos concretos y la práctica jurídica (...) esta puede entonces crear derecho, regular fenómenos culturales que son relevantes para un Estado en un momento histórico determinado" (Rodríguez. 2006). En este sentido está basada en un estudio descriptivo, puesto que su principal objetivo es el análisis de la relación de las propuestas territoriales para la construcción de paz y la erradicación de la pobreza extrema con el PND, y de esta forma diagnosticar el estado de los municipios de San Juan Nepomuceno y el Carmen de Bolívar.

El método que se implementa es el diseño de campo, por medio del cual se analizaran las situaciones que se presenten en los territorios. En este sentido se utilizarán técnicas de recolección de información como la observación, retomada como una estrategia fundamental del enfoque cualitativo, pero a su vez como "un proceso rigurosos de investigación, que permite describir situaciones y/o contrastar hipótesis, siendo por tanto un método científico"(Fernández. 1980, Pág. 135). Esta técnica será orientada a la respuesta concreta de los objetivos específicos. Por tanto, para efectos de esta investigación, será intencionada, selectiva e interpretativa y semi-estructurada, es decir, contará con una guía previamente elaborada que permitirá dar paso o tener acceso a cualquier otra información relacionada con el problema de investigación.

La entrevista como "la comunicación interpersonal establecida entre el investigador y el sujeto de estudio a fin de obtener respuestas verbales a los interrogantes planteados sobre el problema propuesto. Se considera que este método es más eficaz que el cuestionario, ya que permite obtener una información más completa"(Galán. 2009. Pág.1). Esta también será semi-estructurada, con el ánimo de permitir que los (a) sujetos puedan realizar intervenciones de acuerdo a su perspectiva de la materialización del PND en el territorio.

\section{LA ECONOMÍA, EL DERECHO Y EL CONFLICTO}

Puede decirse del derecho que es un conjunto de normas que reglamentan la conducta humana, a partir de las leyes creadas por el congreso de la República, y que son la herramienta principal de la disciplina. No obstante, las leyes por si solas no son imperativas, 
de ser así no sería necesaria la rama judicial. Entonces, ¿Quién hace cumplir las leyes? Aquí está el primer análisis, un jurista respondería de manera automática que los jueces, pues en efecto son quienes la aplican, quienes analizan la conducta y la confrontan con la norma.

Carnelutti (2015) aplica el sentido amplio de la anterior pregunta con un ejemplo sencillo: “(...) Si al pasar por una frutería me vienen ganas de comer una manzana con la tentación de alargar la mano y arrebatarla, pero, en cambio, pago el precio y la compro, aun sin darme cuenta de eso haría el mismo razonamiento que hace el juez, si, habiendo cedido a la tentación, me declarase culpable de hurto (...)" Todo ello para acertar en que los ciudadanos también aplican las leyes cuando omiten o realizan una conducta.

Ahora bien, es sabido que las necesidades de los seres humanos son ilimitadas, aun cuando los bienes son limitados, por ello (Carnelutti, 2015) la propiedad es antes que un fenómeno jurídico, un fenómeno económico. Tan limitación de los bienes, hace que el ser humano tenga en si el deseo de arrebatar, de quitar, el deseo de la invasión del dominio, y los confines entre el haber de una persona y otra se violan, entonces Hobbes (1651) plantea su tesis que "el hombre es un lobo para el hombre", económicamente se comporta como "un animal de presa, y en vez de dejar a cada cual lo que tiene, quiere arrebatárselo", lo que genera el inicio de la guerra, en su sentido general, no solo de los pueblos, sino de la guerra entre los individuos que genera caos.

El truco del derecho radica en que las personas no pueden vivir en el caos, muestra de ello es que en ellas siempre vive la añoranza de la paz, para ello necesitan orden y esta es la raíz del pacto social, o como Hobbes (1651) lo llamó: el contrato social.

Lo que permite razonar en principio que la economía como distribuidor de los bienes limitados siempre va a necesitar del derecho para no permanecer en el caos, y que por ello, no existe cuestionamiento alguno en que el desarrollo no es meramente económico, sino que por el contrario debe ser integral, es decir económico y social.

\section{DESARROLLO INTEGRAL, MÁS ALLÁ DE LA DISTRIBUCIÓN ECONÓMICA}

Generalmente, al mencionar la palabra desarrollo, las personas se remiten a los sistemas industriales en las economías de los países, se identifican condiciones y estructuras socio-económicas para hallar una senda de desarrollo económico sostenido productivo o no (Cerso, 2006), basándose en los tres factores de producción de la economía clásica: el trabajo, la tierra y el capital.

En la economía históricamente se ha intentado desde distintos puntos de vista conceptualizar la pobreza, sin embargo economistas como Adam Smith, se centraban más en el problema de la riqueza y dejaban de lado la investigación científica y rigurosa 
del fenómeno de la pobreza, por lo menos hasta finales de 1940 la economía se dirigía a este enfoque. Después de la segunda guerra mundial y siendo esta desencadenante de nuevas miradas por las distintas problemáticas que surgieron, se empieza a observar en detalle el fenómeno de la pobreza y el subdesarrollo con mayor rigurosidad.

Las definiciones que se le otorgan a la pobreza de aquí en adelante, han sido variadas, ser pobre puede considerarse el no disponer de los recursos para obtener los medios mínimos de subsistencia o puede ser simplemente hambre (Banco Mundial, 2015. p1). Para Sen, la pobreza no es simplemente la falta de ingreso, sino “(...) la falta de capacidades básicas y la forma de obtenerlo"(Sen, 2000, p.117). Amartya, plantea que existe una subjetividad en cada persona que trata de sobrevivir y que ha experimentado la pobreza, por ello afirma la importancia del desarrollo como libertad a la hora de evaluar al individuo, su sociedad y su papel en ella y defiende la abolición de aspectos de la tradición si esos aspectos conllevan graves pérdidas de libertad y pobreza a las personas que lo sustentan.

La desigualdad entre los componentes de la sociedad es lo que genera la pobreza esto haciendo referencia específicamente a la mala distribución de las riquezas. No obstante, en el Plan Nacional de Desarrollo 4 en pro de disminuir la desproporcionalidad creó estrategias transversales: competitividad e infraestructura estratégicas; movilidad social; transformación del campo; seguridad, justicia y democracia para la construcción de la paz; buen gobierno y crecimiento verde, puntos fundamentales todos para lograr disminuir la pobreza. Sin embargo, puede observarse que todas las acciones están dirigidas al desarrollo económico, lejos de un desarrollo integral, que tiene sus pilares en la ampliación de las cinco libertades:

1. Libertades políticas a todas las capacidades de los colombianos para influir en el manejo de su sociedad.

2. Libertades económicas o los derechos de una persona a disfrutar de los recursos económicos y prosperar.

3. Oportunidades sociales o todos los servicios públicos que ofrece una sociedad para la mejora personas de los individuos que la conforman.

4. Garantías de transparencia, que influyen en el grado de confianza que mostrarán los individuos en sus tratos mutuos.

5. Seguridad protectora para ayudar a los desfavorecidos por cualquier causa a sobrevivir y prosperar en sociedad.

Adicionalmente, es evidente que no es posible hablar de la erradicación de la pobreza sin la inclusión de los distintos grupos poblacionales (campesinos, afro descendiente, indígenas, etc.) que permitan la participación y las libertades políticas, tampoco sin libertades económicas, sin oportunidades, sin garantías de transparencia y sin seguridad protectora. La historia ha demostrado que la consecución de una paz sostenible está muy

${ }^{4}$ En adelante PND. 
relacionada con la eliminación de la pobreza, puesto el conflicto interno en Colombia es una particularidad que no permite conseguir un país sin pobreza por todos los efectos colaterales del mismo y las implicaciones que este genera, por lo que encierra la problemática en un círculo vicioso que no presenta salida alguna.

Las problemáticas presentes en el país por años como el hambre, la desnutrición, enfermedades por insalubridad y por falta de medios para evitarlas o tratarlas, la falta de libertad política, la denegación de los derechos humanos, la desigualdad entre hombres y mujeres, entre otras (Sen, 2004) representan el más bajo nivel del desarrollo integral, porque permiten proporcionar el bienestar social, y si este es invisible, no se puede hablar de una oportuna distribución de los bienes, mucho menos de participación, de seguridad protectora, libertad política o económica, por el simple hecho que no puede existir una libertad económica para elegir en que invertir o gastar recursos cuando no existen recursos. Todas las libertades complementan entre sí como medios para conseguir el desarrollo, no pueden ser aisladas una de la otra. El crecimiento económico además de contemplarse como un medio para incrementar la renta privada, también puede contemplarse como refuerzo para las libertades y en consecuencia el incremento del bienestar social o jurídicamente el bien común.

Es indudable que el desarrollo integral no puede entenderse con tal si no es un desarrollo como libertad, como "(...) un proceso de expansión de las libertades reales de las que disfrutan los individuos" (Graziano, 2002, p.1). Lo que supone, que no es posible medir el desarrollo con otro indicador que no sea el aumento de las libertades de los individuos" ya que esta es el fin del desarrollo.

Las libertades también son el medio, “(...) además de ser el objetivo último del desarrollo, la libertad puede ser una excelente herramienta para lograr el mismo. Se trata del papel instrumental de la libertad en el desarrollo" (Graziano, 2002, p.1). Por ello se refiere directamente a los derechos y a la confluencia de los distintos tipos, y argumenta que el aumento de la libertad en el ser humano, fomenta el desarrollo, y que la eficacia de la misma esta en interrelacionar los distintos tipos de libertad. Sen, dentro de su tesis, se apoya en Aristóteles, por lo que entiende la riqueza no como un fin en sí mismo, sino en las cosas que se puedan hacer con ella.

\section{LA TEORÍA DEL DESARROLLO EN EL PLAN NACIONAL DE DESARROLLO (PND)}

Determinar cuáles son los principales retos que tiene un nuevo mandatario presidencial puede ser más complicado de lo que parece y más siendo el presidente de un país diverso y con complejos problemas sociales, económicos y políticos, por eso se hace necesario tener una hoja de ruta que permita direccionar la inversión del gobierno nacional partiendo de las necesidades de los habitantes, esta hoja de ruta es el Plan Nacional de Desarrollo. 
El PND es el documento que sirve de base y provee los lineamientos estratégicos de las políticas públicas formuladas por el Presidente de la República a través de su equipo de Gobierno. Su elaboración, socialización, evaluación y seguimiento es responsabilidad directa del Departamento Nacional de Planeación. Es el instrumento formal y legal por medio del cual se trazan los objetivos del Gobierno permitiendo la subsecuente evaluación de su gestión.

El Plan Nacional de Desarrollo 2015- 2018 lleva por nombre "todos por un nuevo país" tiene como propósito generar un país en: Equidad, Paz y fortalecido en la educación, aspectos que para el gobierno nacional garantizan la sostenibilidad del pueblo colombiano y que llevaría a los niveles de calidad de vida que merece este país, siendo estos tres pilares un circulo virtuoso para el desarrollo.

Una sociedad en paz puede focalizar sus esfuerzos en el cierre de brechas y puede invertir recursos en mejorar la cobertura y calidad de su sistema educativo. Una sociedad equitativa en donde todos los habitantes gozan de los mismos derechos y oportunidades permite la convivencia pacífica y facilita las condiciones de formación en capital humano. Finalmente, una sociedad educada cuenta con una fuerza laboral calificada, que recibe los retornos a la educación a través de oportunidades de generación de ingresos y de empleos de calidad, y cuenta con ciudadanos que resuelven sus conflictos sin recurrir a la violencia. De forma más simple: la paz favorece la equidad y la educación, la equidad propicia la paz y la educación, y la educación genera condiciones de paz y equidad. Por ello, este Plan Nacional de Desarrollo se concentra en estos tres pilares, fundamentales para la consolidación de los grandes logros de los últimos cuatro años y para el desarrollo de las políticas para los próximos cuatro, en pro de un nuevo país (PND, 2014, p. 29).

Dentro de las principales misiones que tiene el gobierno nacional y el cual está reflejado en el plan nacional de desarrollo es disminuir las brechas de desigualdad y empezar a utilizar las potencialidades de los ciudadanos colombianos de esta manera construir bases solidad que permitan un desarrollo integral hacia el post conflicto, en el capítulo Colombia equitativa y sin pobreza extrema se plantean diferentes objetivos y lineamientos que tienen como columnas vertebrales programas y proyectos para alcanzar estar metas.

Dentro de los principales se encuentran: diversificar y sofisticar apuestas productivas; potenciar la contribución de la tecnología y la investigación en desarrollo de iniciativas productivas; fortalecer la competitividad agropecuaria para consolidar el campo como generador de empleo y riqueza para los habitantes rurales. Todo dirigido al terreno económico.

Pero, para hablar del desarrollo de una sociedad hay que analizar la vida de quienes la integran, no puede considerarse que hay éxito económico sin tener en cuenta la vida de los individuos que conforman la comunidad. El desarrollo es entonces el desarrollo de 
las personas de la sociedad. Por este motivo define concretamente: "El desarrollo es un proceso de expansión de las capacidades de que disfrutan los individuos" (Sen, 2000, p. 123).

Si bien se advierte que la palabra capacidad no es atractiva en sí misma, que se ha usado para hablar de las tierras y no de las personas, de lo productivo y no de lo virtuoso, no es menos cierto que las capacidades simbolizarían las posibilidades que tienen los individuos de alcanzar desempeños valiosos, es algo más allá de lo palpable, de lo medible, va más lejos de lo que se reconoce como capital humano, si bien ambos conceptos centran su atención en el hombre, la diferencia principal a tener en cuenta está dada porque la literatura económica sobre capital humano pone mayor énfasis en el rol del ser humano como productor de bienes y servicios, por lo que es primordial observar y estudiar cómo cada mejora en la calificación del hombre hace que éste sea más productivo. En cambio, el punto de vista de la capacidad humana centra su atención en la capacidad de los individuos para vivir la vida, y por ello se analizan los motivos que éstos poseen para valorar y aumentar las alternativas reales entre las cuales poder optar.

Cada persona, en función de sus características, origen y circunstancias socioeconómicas con las que convive, entre otros aspectos, tiene la capacidad para hacer ciertas cosas que valorará por diferentes motivos. Tal valoración puede ser directa o indirecta:

- La primera está en función de aquellos elementos que implican que podrá enriquecer su vida, es decir que le permitirán tener una mejor calidad de vida, como estar bien nutrido o sano.

- La segunda tiene que ver con la posibilidad de contribuir más y mejor en la producción. De esta manera, la perspectiva de la capacidad humana es más abarcaría que la teoría del capital humano, esta última es una expresión más limitada de la capacidad humana.

El pilar de la educación es fundamental para el desarrollo integral y el PND lo reconoce al incluirla como uno de los tres centros de su eje, implica que a medida que las personas reciben educación y aumentan su capacidad de lectura y escritura puede ser mucho más importante el efecto generado sobre la calidad de vida de la población, lo que indica que por medio de una mejora en la educación y en otras variables que tengan más que ver con el nivel de riqueza de una sociedad. La educación permite el progreso del país, en casos como Japón, China y Corea del Sur se ha visto que la inversión en educación básica tuvo resultados positivos para el progreso; es difícil participar de la economía mundial exitosamente si la gente no sabe leer y escribir, producir de acuerdo a las especificaciones o tener control de calidad, entre otras consideraciones.

Por medio de la educación pueden incentivarse actitudes favorables al desarrollo, por ejemplo puede acrecentarse el diálogo social, promoviéndose la estabilidad y la paz 
dentro de la comunidad, las cuales son deseables por sí mismas, pero además crean un clima favorable para el desarrollo. Lo que permite visionar la importancia de los centros educativos para la economía y la sana convivencia. La influencia de la escuela incentiva la identidad de la persona, la forma en que la persona se observa a sí misma y a los otros. Por ello debe estar disponible para todos, para que los niños reciban ideas de diferentes marcos o puntos de vista, y sean animados a pensar por ellos mismos y con fundamentos. La educación es una capacidad esencial. Cuando la persona es analfabeta su habilidad para entender e invocar sus derechos es limitada, y su falencia educacional puede liderar otra clase de privaciones. Mientras que la educación básica contribuye a reducir la privación básica, la falta de educación tiende a hacer persistir el problema para las personas que están en los estratos sociales más bajos, cuyos derechos no son respetados porque ellos no tienen la habilidad de leer, observar y demandar, ni saben cómo hacerlo. Así, la diferencia educacional se relaciona con la diferencia de clases, quienes no tienen la posibilidad de ir a la escuela, extrañamente tienen voz en política para hacer sus demandas.

Al mismo tiempo, la educación permite al individuo socializarse e intercambiar ideas y obtener un empleo, mejorar las condiciones de calidad de vida, ayuda a aumentar la libertad y disminuir la inseguridad personal, asociada con la social. Las desigualdades en acceso, inclusión y logros escolares conducirán a diferencias en habilidades, que a su vez diferencian la participación de los individuos en el mundo contemporáneo. "Si continuamos dejando a gran parte de la población mundial fuera de la órbita de la educación, vamos a construir un mundo, no solo menos justo, sino también menos seguro" (Sen, 2000, p. 123).

Se debe resaltar su rol importante en la salud, asociada con la disminución de las epidemias. Esta afirmación se sustenta en estudios que reflejan que la educación básica posee mayor impacto que la educación especializada en salud.

Las libertades como capacidades fundamentales son las diferentes combinaciones de funciones que el individuo puede conseguir y que le permiten lograr distintos estilos de vida. Por ejemplo, una persona rica que ayune puede conseguir los mismos resultados funcionales en lo que se refiere a comer o nutrirse que una persona desfavorecida que se vea obligada a pasar hambre, pero la primera tiene un 'conjunto de capacidades' diferente al de la segunda (la primera puede decidir comer bien y estar bien nutrida, mientras que la segunda no), lo que hace que las condiciones varíen, en torno a las capacidades adquiridas.

Dentro de los determinantes de las libertades del hombre menciona las instituciones sociales y económicas, como por ejemplo los servicios de educación y salud, o los derechos políticos y humanos, tales como la libertad de expresión y el derecho a elegir las autoridades públicas. "Las libertades políticas (en forma de libertad de expresión y 
elecciones libres) contribuyen a fomentar la seguridad económica. Las oportunidades sociales (en forma de servicios educativos y sanitarios) facilitan la participación económica. Los servicios económicos (en forma de oportunidades para participar en el comercio y la producción) pueden contribuir a generar riqueza personal y general, así como recursos públicos para financiar servicios sociales" (Sen. 2000). El hecho de que existan libertades y derechos políticos, incluida la libertad de expresión, hace más fácil evitar desastres económicos como las hambrunas.

\section{LA EDUCACIÓN COMO EJE CENTRAL}

Según Amartya Sen hay pruebas suficientes de que, aun con una renta baja, un país que logre que todos sus ciudadanos accedan a la educación y a la asistencia sanitaria, puede obtener muy buenos resultados en cuanto a longevidad y calidad de vida de toda la población. (Sen, 2000, p. 156). No ser capaz de leer, escribir, contar o comunicarse es una tremenda privación y constituye un caso extremo de inseguridad, ya que el individuo tiene la certeza de la privación y la ausencia de cualquier chance de evitar ese destino.

Si bien la riqueza es la manera de alcanzar una calidad de vida o el medio mediante el cual los individuos la obtienen, también es necesario detectar y tener en cuenta sus limitaciones. El aumento del producto no puede considerarse como único determinante. Éste es tan solo un medio primordial para expandir las libertades de los individuos que viven en sociedad, aunque no el único factor que influye. Cabe resaltar que la riqueza se manifiesta en que permite al individuo conseguir libertades fundamentales.

Es preciso resaltar que en el territorio colombiano se carece de ciertas libertades y que la verdadera razón por la cual aún no hemos crecido de la forma como se ha proyectado es por la necesidad de muchos dirigentes de mantenernos atados, por el arrebato de los bienes que al final ha terminado en un caos social.

La libertad es esencial para el proceso del desarrollo por dos razones diferentes. 1) La razón evaluativa: La valoración del progreso debe hacerse tomando en cuanta principalmente si mejoran las libertades que tiene la gente. 2) La razón efectividad: La consecución del desarrollo está completamente subordinada al libre albedrío de la gente. Con anterioridad he señalado la primera motivación, la razón evaluativa para enfocarnos en la libertad. Al ir en pos de la otra, la de la efectividad, tenemos que fijarnos en las conexiones empíricas relevantes, en particular las conexiones que refuerzan entre sí las libertades de diversas clases. Es a causa de estas interconexiones que el libre y sustentable albedrío emerge como un importante motor del desarrollo. No sólo es el libre albedrío en sí una parte "constitutiva" del desarrollo, sino que también contribuye al fortalecimiento de libre albedrío de otras clases (Sen, 2009, p. 125). 
El ingreso del (PNB) o de los ingresos individuales, puede desde luego, representar un medio muy importante para ampliar las libertades que disfrutan los miembros de la sociedad, sin embargo, esas libertades dependen también de otros factores, como los planes sociales y económicos (programas para la educación y el cuidado de la salud) y los derechos civiles y políticos (libertad en participar en el debate y escrutinios públicos). De la misma forma, la industrialización, el progreso tecnológico o la modernización social pueden contribuir sustancialmente a la expansión de la libertad humana, pero esta depende de otros factores y no debe aparecer como única apuesta para el desarrollo.

\section{CONCLUSIONES}

Colombia como país democrático influenciado por una fuerte influencia del modelo económico capitalista y neoliberal, no puede desconocer el fin principal del Estado: el bien común. Aun cuando la empresa privada este presente y permee las dinámicas públicas no puede el objetivo de esta convertirse en el modelo de desarrollo que se busca, puesto que este debe ir más allá del límite económico.

Colombia es un país que a pesar de sus riquezas naturales, agrícolas y metalúrgicas sigue siendo un estado con personas en extrema pobreza y con altos índice de desempleo, las estrategias del estado apaciguan de manera general la calidad de vida de los ciudadanos y a pesar de que factores económicos como la inflación estén aumentando y las acciones ya se empiecen a sentir en el caso de los aumentos de la tasa de interés, las medidas son pequeños paños de agua tibia.

Es valioso el crecimiento económico desde los aspectos que tradicionales, dándole prioridad a la industria antes que al ser como tal y a su necesidad de encontrar libertades que lo lleven a ser humanamente más productivo por lo que no es posible hablar de una motivación a la felicidad. Pero con esto no se quiere decir que solo la felicidad llevara al desarrollo económico es fundamental tener en cuenta que un desarrollo sostenible debe ser económico y social.

El bienestar social debe ser en todo momento analizado desde el desarrollo como mejora en la calidad de vida, no solo como viven las personas, sino también que alternativas tienen las mismas, se tiene en cuenta el control que las personas tengan de sus vidas, las capacidades adquiridas que les permitan tener las libertades de elegir. Al hacer referencia del control que tiene los individuos sobre sus vidas, se hace estudia al termino de libertad en el sentido de no privación o ausencias de restricciones.

Las libertades juegan un papel indispensable en el desarrollo económico de los países, puesto que estas, permiten a su vez el crecimiento económico y lo que podríamos denominar crecimiento social. Para argumentar su postura, cita un dato clave en su obra desarrollo y libertad: “(...) ningún país democrático independiente a sufrido una 
hambruna, y es que la libertad política de los ciudadanos se puede traducir en un cambio en el gobierno si el presente no lo hace bien, lo que en última instancia puede obligar a los gobernantes a hacer todo lo posible para garantizar el abastecimiento de alimentos" (De Sol citando a Sen, 2004). Es por lo anterior, que para Sen, aunque todas las libertades son importantes, existe prioridad de una sobre las otras, sobre todo las que nos permiten decidir el futuro de un país.

Se concluye en términos generales, las grandes teorías del desarrollo se refieren a condicionantes más relacionados con los ámbitos internacionales o mundiales, o bien nacionales como es el caso objeto de estudio de nuestra investigación sobre desarrollos económicos y sociales para la búsqueda de la igualdad y erradicación de la pobreza extrema. Los componentes centrales del desarrollo humano enfatizan características que ese desarrollo debe tener, es frecuente encontrar en todo nuestro estudio como eje principal el aspecto económico y la relación con conceptos amplios como la pobreza y el desarrollo humano; de enfoque pone el énfasis en el ser humano y en su libertad, libertad como individuo en una sociedad, siendo este su principal medio de desarrollo substánciales, pone en el centro no solo los medios, sino también los fines del mismo. Se deja para estudios posteriores el análisis detallado de como concebir al desarrollo como un proceso de expansión de las libertades fundamentales.

\section{REFERENCIAS BIBLIOGRÁFICAS}

Bases del plan de desarrollo todos por un nuevo país. Colombia equitativa y sin pobreza extrema. Encontrado en: https://colaboracion.dnp.gov.co/CDT/Prensa/ Bases\%20Plan\%20Nacional\%20de\%20Desarrollo\%202014-2018.pdf. Acceso: 14 de Noviembre de 2015.

Banco Mundial. definiciones de los objetivos de desarrollo del milenio [En Línea] [http:// www.bancomundial.org/temas/omd/definiciones.htm] acceso [ 05, octubre, 2015]

Carnelutti, Francesco (2015). ¿Cómo nace el derecho? Editorial: Temis. 4ta impresión. Bogotá-Colombia.

Cerso, Fultado (2006). Teoría política y desarrollo económico. Encontrado en: http:// books.google.es/books?id=7Rpew CLu8C\&printsec=frontcover\&source=gbs_ge_ summary_r\&cad=0\#v=onepage\&q\&f=falseAcceso: 30 de agosto de 2015. México.

Del Sol, Jose Gregorio (2004). Energía, economía y desarrollo sostenible. Capítulo 4. Citando a SEN. Amartya. Desarrollo y libertad.

Fernández, Ballesteros (1980). Métodos de investigación en educación especial. [Artículo de internet] Link: https://www.uam.es/personal_pdi/stmaria/jmurillo/ 
InvestigacionEE/Presentaciones/Curso_10/Observacion_trabajo.pdf. Acceso: 26 de mayo de 2016.

Galán. Amador. Metodología de la investigación. [Artículo de internet]. Link: http:// manuelgalan.blogspot.com.co/2009/05/la-entrevista-en-investigacion.html. Pág. 1. Acceso: 26 de mayo de 2016.

Graziano, Ricardo (2002). Amartya Sen y el desarrollo como libertad. La viabilidad de una alternativa a las estrategias de promoción del desarrollo. Departamento de Ciencia Política y Gobierno Licenciatura en Estudios Internacionales. Universidad Torcuato Di tella. Encontrado en: http://www.colombiaaprende.edu.co/html/ docentes/1596/articles-346050_recurso_3.pdf. Acceso: 30 de agosto de 2015.

Hobbes, Thomas (1651). Leviatan. Publicaciones Cruz. O.S.A. Reimpresión de 1999. México D.F.

Rodríguez, Bartolo Pablo (2006). Metodología jurídica. México. D.F. Osford University. Primera edición. Séptima impresión. Libro virtual. Link: http://www.libroesoterico. com/biblioteca/metafisica/Metodologia\%20Juridica-Bartolo-Pablo-RodriguezCepeda.pdf.

Sen, Amartya. El desarrollo como libertad. México: D - Instituto Nacional de Ecología, 2009. ProQuestebrary. Web. 24 November 2015.

Copyright (C) 2009. D - Instituto Nacional de Ecología. All rights reserved. 